\title{
Analysis of one-way and two-way street configurations on urban grid networks
}

\section{Working Paper}

\section{Author(s):}

Ortigosa, Javier; Gayah, Vikash V.; Menendez, Monica

Publication date:

2014

Permanent link:

https://doi.org/10.3929/ethz-b-000089504

Rights / license:

In Copyright - Non-Commercial Use Permitted

Originally published in:

SVT Working Paper 75, https://doi.org/10.3141/2491-02 


\title{
Analysis of one-way and two-way street configurations on urban grid networks
}

\author{
Javier Ortigosa ${ }^{1}$ \\ Institute for Transport Planning and Systems, ETH Zurich \\ Vikash V. Gayah \\ Civil and Environmental Engineering, The Pennsylvania State University \\ Monica Menendez \\ Institute for Transport Planning and Systems, ETH Zurich
}

\begin{abstract}
The design of urban street networks is a subject of much controversy because there is no consensus on the best way to organize streets to maximize mobility. Debates between one-way and two-way street networks have existed since cars were introduced into urban environments and are still ongoing. In this paper, we provide additional insights on this issue by analyzing traffic behavior on three different street configurations: one-way streets, two-way streets, and two-way streets with prohibited left turns. The outcomes of this paper will furnish urban planners and policy makers with a better understanding of the mobility provided by each of these types of urban street network layouts.
\end{abstract}

This study is focused on abstract finite grids operating in both uncongested and congested conditions. Analytical formulations are created and used to compare the three network types under low demand scenarios when the network is uncongested. These formulations rely on a fixed traffic assignment scheme in which drivers are assumed to minimize their overall travel distance and number of turning maneuvers. They are used to reveal general insights. In addition, simulation techniques are employed with a static traffic assignment algorithm to examine the behavior of networks under more realistic congested situations.

In general, the study reveals that two-way networks provide the shortest distance traveled and result in more uniform congestion patterns due to route redundancy. However, they are severely penalized by restrictions in capacity at the intersections due to more complicated phasing schemes, which leads to higher average travel times when the network is large, has short links, or is lightly congested. Oneway networks offer the longest travel distances but have intersections with very high capacity. They perform better when the network is very large or links are very short. Finally, two-way networks with prohibited left turns provide the best compromise between distance traveled and intersection capacity, which makes them suitable for many different scenarios with low and medium congestion. Their main weakness is that they suffer from inhomogeneous congestion patterns caused by a lack of route redundancy. This could lead to very large travel times when the network is very congested.

Keywords: urban traffic - urban network design - grid - one-way streets - two-way streets - prohibited left turns

\footnotetext{
${ }^{1}$ Corresponding author: javier.ortigosa@ivt.baug.ethz.ch / +41446339415
} 


\section{Introduction}

\section{Which design better accommodates car traffic in urban areas: a one-way or two-way street network?}

Planners and policy makers are often tasked with deciding between one-way and two-way streets in urban transportation networks, particularly when making the choice to change a street's configuration. There are many factors that go into this decision including: safety, urban design, livability, and traffic operations. However, to date no consensus has been reached on the optimal network configuration. In this paper, we try to provide additional insights on this controversial issue by focusing specifically on how street network configuration impacts car traffic operations in grid networks.

The way cities are planned and streets organized influences our everyday mobility. In the past, traffic engineers tended to increase the amount of roadway infrastructure to keep up with growing demands. However, this leads to even greater car demands, negative car externalities and even more urban space dedicated for cars (e.g. Cervero, 2001). More recently, however, traffic engineers and urban planners have shifted focus to developing more efficient urban structures to reduce these negative impacts and revert this cycle. A more efficient network is that one able to provide a higher mobility while employing fewer resources, in this case urban road space dedicated to roadways. Despite these advances, we believe that there is still not a common agreement in how to configure streets in the most basic of urban structures - a grid. This fact has motivated the present paper.

The topic is gaining particular interest in the U.S. where grid networks are common and there has been a recent movement to convert streets from one-way to two-way operation (Walker et al., 2000). This movement has awakened a research debate with conflicting views on which configuration is better (e.g. Lyles et al., 2000). Walker et al. (2000) advocates for the conversion to two-way networks based on the changes in the character of downtown areas (denser cities, mixed-use activities, intermodal transportation). In addition, there are many safety-oriented studies that defend or criticize either one approach or the other (e.g. Tindale and Hsu, 2005; Hocherman et al., 1990; Stemley, 1998). In favor of one-way streets, there is the argument that their intersections have fewer conflict points. On the other hand, two-way streets are thought to induce lower vehicle speeds and hence lower risk of accidents and their associated impacts. Finally, some (e.g. Walker et al., 2000) also advocate that two-way streets are better for business, as they increase the 'livability' in the neighborhood. To the authors' knowledge, this debate does not seem to be very relevant in Europe. As a matter of fact, well-known city grids like Barcelona (Spain), Glasgow (UK), or Mannheim (Germany), are primarily composed of one-way streets. The difference perhaps is that European cities have denser urban centers with mixed activities as a result of many years of city evolution without automobiles. Hence, many urban centers maintain the same street structures from centuries ago. That is an advantage for one-way streets, since some streets might only devote one lane to the car movement.

Strictly focusing on the movement of cars, two-way streets provide a higher accessibility but also offer less capacity at intersections. On the other hand, one-way street networks provide intersections with higher capacities and travel speeds, but force drivers to travel longer routes on average. This trade-off has received little quantitative attention in the literature and is the focus of this present paper. Walker et al. (2000) qualitatively discuss the extra distance and movements required in a one-way street network, but quantitative metrics and details are not provided. Meng and Thu (2004) analyze operations after the conversion of a two-way grid into a one-way grid with a CORSIM simulation. This study concludes that one-way streets perform better than two-way streets mainly because of the higher average travel speeds observed. However, higher average speeds do not necessarily mean that 
people get to their destinations faster if they have to travel longer distances. To overcome this gap, Gayah and Daganzo (2012) examine the maximum rate at which trips could be completed in various grid networks, including one-way, two-way, and two-way with prohibited left turns. Using this new metric, this study is the first to quantify the potential trade-offs between additional distance traveled and capacity available at intersections by combining them into a single metric: trip-serving capacity. The study shows that two-way streets networks outperform one-way street networks when trips are long, and two-way street networks with prohibited left turns always outperform one-way street networks. However, the work uses abstract networks of infinite size and optimistically assumes that congestion is evenly distributed across the network. Additionally, the metric proposed only considers capacity flows and cannot be used to provide insights into operations during either free-flow or congested conditions. Boyles et al. (2014) employ simulation techniques to study abstract grid networks under different levels of congestion. However, the authors focus primarily on developing a static traffic assignment model that is able to represent the low-conflict intersections maneuvers of Eichler et al. (2012) innovative networks, and do not emphasize the differences between one-way vs. two-way street networks.

The present paper tries to fill the gaps in the literature by comparing directly one-way and two-way networks (including those with prohibited left turns) under more realistic traffic conditions. We employ an abstract grid but with a finite size where the border, network size, and link lengths, all play a role. Analytical formulations are employed to study the case of low traffic demands in which congestion patterns do not significantly influence operations. These formulae provide direct insights on the trends that should be expected with each configuration and allow low demand scenarios to be analyzed simply. Moreover, we look at the effects of inhomogeneous congestion patterns that arise in realistic networks through simulations to study the behavior of congested networks. These simulations rely on a static traffic assignment that mimics drivers' tendencies to route themselves to minimize their own personal travel time.

Finding an optimal and general solution for organizing traffic in cities is nearly impossible since every city has its own particularities. However, each network layout has advantages and disadvantages and urban and traffic planners should be aware of them before carrying out any action on the city's layout. The conclusions of this paper provide urban and traffic planners a better understanding of the effects of each network layout on traffic, as well as a better idea of the conditions under which each network type should perform best. Increasing this awareness and understanding is the main goal of this paper.

The rest of the paper is organized as follows. First, we introduce analytical formulations to describe operations on uncongested networks. Second, we present the simulation model used to analyze grids in congested scenarios. Finally, we analyze the results and present the main conclusions of this research.

\section{Uncongested analysis: analytical formulations for finite grids}

We consider here an abstract, square grid network that has $n$ intersection ${ }^{2}$ nodes per side $\left(n^{2}\right.$ nodes in total). The origins and destinations of trips (i.e. demand nodes) are located in the midpoint of every link; to emulate the generation and attraction of trips along the street. The demand is uniform, which means that every demand node exchanges the same amount of trips, $T$, with all the other demand nodes. Figure 1 shows the three kinds of network configurations we analyze: two-way streets with all the turns allowed (TW), two-way streets with prohibited left turns (TWL), and one-way streets (OW).

\footnotetext{
${ }^{2}$ Note that also nodes on the perimeter, including the corners, are considered as intersection nodes.
} 
$T R_{T W}=T \cdot n \cdot(n-1)^{2} \cdot(3 n-2)$.

The total number of trips made in the network, $N$, was also determined by calculating the number of trips exchanged between each pair of demand nodes:

$N=2 \cdot T \cdot n \cdot(n-1) \cdot\left(2 n^{2}-2 n-1\right)$.

The ratio of (1) to (3) yields the average distance traveled (i.e. average trip length), $A D_{T W}$ :

$A D_{T W}=\frac{1}{3} \cdot \frac{\left(4 n^{3}-6 n^{2}+3 n-2\right)}{\left(2 n^{2}-2 n-1\right)} ;$

similarly, the ratio of (2) to (3) yields the average number of turns per trip, $A T_{T W}$ :

$A T_{T W}=\frac{(n-1) \cdot(3 n-2)}{\left(2 n^{2}-2 n-1\right)}$. 
$T D_{T W L}=\frac{8}{3} \cdot T \cdot n \cdot\left(n^{4}-n^{3}-\frac{9}{4} n^{2}+\frac{13}{4} n-1\right)$, and

$T R_{T W L}=4 \cdot T \cdot n \cdot(n-1)^{2} \cdot(2 n-1)$.

$A D_{T W L}=\frac{4}{3} \cdot \frac{\left(n^{4}-n^{3}-\frac{9}{2} n^{2}+3 n-1\right)}{(n-1) \cdot\left(2 n^{2}-2 n-1\right)}$

similarly, the ratio of (7) to (3) yields the average number of turns per trip, $A T_{T W L}$ :

$A T_{T W L}=\frac{2 \cdot(n-1) \cdot(2 n-1)}{\left(2 n^{2}-2 n-1\right)}$

Since the total number of trips made, $N$, remains the same, the ratio of (6) to (3) yields the average trip length, $A D_{T W L}$ :
10

\subsection{One-way streets (OW)}

There are different possible configurations of OW grids depending on the size of the network and street direction pattern. However, we focus only on those in which trips between any pair of demand nodes can be feasibly made. This leaves only square grids with an even number of intersection nodes per side, and those in which perimeter streets form a traversable loop.

Under these conditions, the total distance traveled, $T D_{O W}$, and the total number of turns made, $T R_{O W}$, in these one-way networks are:

$T D_{\text {OW }}=\frac{2}{3} \cdot T \cdot\left(4 n^{5}+2 n^{4}-\frac{9}{2} n^{3}-29 n^{2}+2 n+24\right)$, and

$T R_{O W}=T \cdot(n-1) \cdot\left(10 n^{3}-6 n^{2}-12 n-8\right)$.

Likewise, since the total number of trips made, $N$, remains the same; the ratio of (10) to (3), and (11) to (3), provide the average trip length, $A D_{O W}$, and the average number of turns per trip, $A T_{O W}$, respectively:

$$
\begin{aligned}
& A D_{O W}=\frac{1}{3} \cdot \frac{\left(4 n^{5}+2 n^{4}-\frac{9}{2} n^{3}-29 n^{2}+2 n+24\right)}{n \cdot(n-1) \cdot\left(2 n^{2}-2 n-1\right)}, \text { and } \\
& A T_{O W}=\frac{\left(5 n^{3}-3 n^{2}-6 n-4\right)}{n \cdot\left(2 n^{2}-2 n-1\right)} .
\end{aligned}
$$




$$
\begin{aligned}
& A D_{T W}>\frac{2 n-1}{3}, \\
& A D_{T W L}<\frac{2 n+2}{3}, \text { and } \\
& A D_{O W}>\frac{2 n+5}{3} .
\end{aligned}
$$

Notice that these lines ((14), (15), (16)) provide lower bounds for TW and OW networks, and an upper bound for TWL networks. As Figure 2 shows, these bounds are fairly tight and can be used as a good approximation for the average trip length, particularly when the network is large. These equations also reveal that trips in a TWL network are at most one block longer than in a TW network, and trips in an OW network are at least two blocks longer than trips in a TW network. These values also correspond well to those derived for infinitely large networks in Gayah and Daganzo (2012).
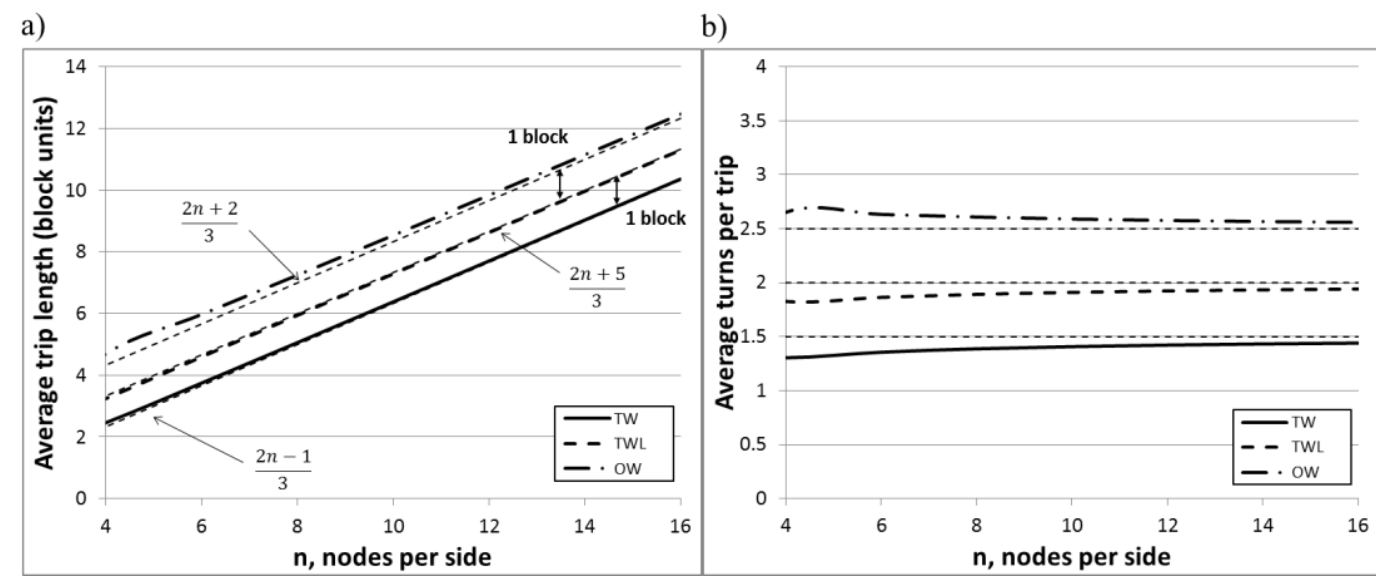

Fig 2. a) Trends for average trip length and, b) Trends for average number of turns per trip.

Similarly, as Figure $2 \mathrm{~b}$ shows, the average number of turns per trip tends to 1.5 in the case of TW networks, 2 for the case of TWL networks, and 2.5 for the case of OW networks. The latter is a lower bound while the first two are upper bounds. Thus, on a one-way network, at least 1 extra turn per trip is required in comparison to a two-way network, and approximately 0.5 extra turns are required in comparison to a two-way network with prohibited left turns.

\subsection{Travel time on networks}

The analytical formulas derived in this section do not provide direct information on travel times. However, we use them to obtain an approximate expression of travel times for uncongested scenarios. To do so, we assume that: 1) cars travel at free flow speed on all links; and, 2) intersections are undersaturated and delays during single cycle can be calculated using a deterministic queuing diagram.

\footnotetext{
${ }^{3}$ As stated earlier, $n$ is the number of intersection nodes per grid side
} 
Adel $=\frac{R^{2}}{2 \cdot C \cdot(1-\lambda / \mu)}$.

4 When the system is very uncongested, the volume to capacity ratio (V/C), $\lambda / \mu$, is very small and can 5 be neglected. Since the average trip length expressions are given in units of block lengths traveled, 6 they also correspond to the number of intersections a car traverses through on its trip. The delay experienced at the intersection depends on the movement being performed. Through and right turn

The travel time on any one link is then $l / v_{f}$, where $l$ is the link length and $v_{f}$ is the free flow speed. Assuming a uniform rate of vehicle arrivals to all intersections, $\lambda$, red time, $R$, cycle time, $C$, and saturation flow, $\mu$, the average delay at any single intersection is: movements (also left turn movements in OW networks depending on the intersection) share the same capacity so they have the same delay associated. However, left-turning vehicles on TW networks experience longer delays because there is generally less green time available for this movement. Since half of all turns on TW networks can be expected to be left turns, the average travel time for TW networks, $A T T_{T W}$, is:

$A T T_{T W}=\left(A D_{T W}-\frac{1}{2} A T_{T W}\right) \cdot \frac{R_{t r}^{2}}{2 \cdot C}+\frac{1}{2} A T_{T W} \cdot \frac{R_{l e}^{2}}{2 \cdot C}+A D_{T W} \cdot \frac{l}{v_{f}}$,

where $R_{t r}$ is the red phase for through and right turn movements and $R_{l e}$ the red phase for left turn movements. For TWL and OW, the expressions are simpler because there is only one red phase, $R$, that is equal in both cases:

$$
\begin{aligned}
& A T T_{T W L}=A D_{T W L} \cdot\left(\frac{R^{2}}{2 \cdot C}+\frac{l}{v_{f}}\right), \text { and } \\
& A T T_{O W}=A D_{O W} \cdot\left(\frac{R^{2}}{2 \cdot C}+\frac{l}{v_{f}}\right) .
\end{aligned}
$$

Figure 3 depicts this average travel time for two link lengths, $100 \mathrm{~m}$ and $200 \mathrm{~m}$, and different network sizes. The street length (i.e. the block size) is also an important factor to consider. Among cities with urban grid structures, street lengths can vary widely. Some measures of U.S. grids (Nairn, 2010) show this variability: Carson City, $230 \mathrm{ft}(\sim 70 \mathrm{~m})$; Portland, $260 \mathrm{ft}(\sim 79 \mathrm{~m})$; Houston, $330 \mathrm{ft}(\sim 101 \mathrm{~m})$; Austin, $350 \mathrm{ft} \mathrm{(} \mathrm{107} \mathrm{m);} \mathrm{Sacramento,} 410 \mathrm{ft}$ ( 125 m); Columbus, 480 ( 146 m); or Salt Lake City, $780 \mathrm{ft}(\sim 238 \mathrm{~m})$. In this paper, both lengths examined $(100 \mathrm{~m}$ and $200 \mathrm{~m})$ are representative of real block sizes.

TW and TWL networks perform similarly for small size networks. However, as soon as the trips become larger (i.e. the networks have more nodes per side), TW networks lose competitiveness in comparison to TWL networks. OW networks slightly improve their travel times in relation to the other network types when network size increases; however, they still always appear to perform the worst, especially when links are longer. Figure 3 also shows how TW networks gain an advantage when links are longer because the time spent overcoming distance gains more influence in the total travel time compared to the time spent delayed at intersections. 
a) Link length $=100 \mathrm{~m}$

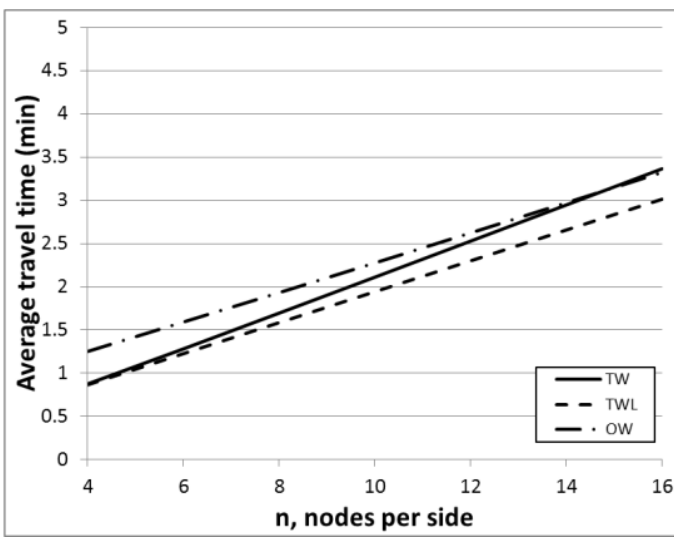

b) Link length $=200 \mathrm{~m}$

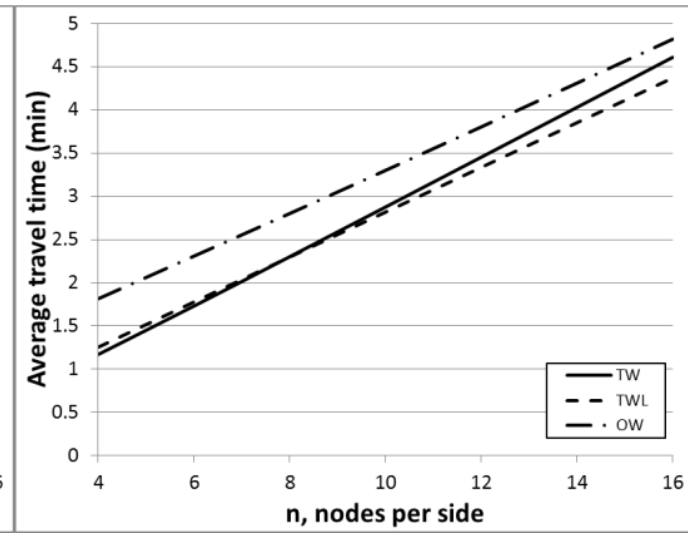

Fig 3. Average travel time according to (18), (19), and (20) for: a) $100 \mathrm{~m}$ link length; and b) $200 \mathrm{~m}$ link length. We considered for TW a $C=60 \mathrm{~s}, R_{t r}=22.5 \mathrm{~s}$, and $R_{l e}=2.5 \mathrm{~s}$; and for TWL and OW, a $C=60 \mathrm{~s}$, and $R=27.5 \mathrm{~s}$ (more information on these values can be found in Section 3).

\section{Congested scenarios: network simulation}

In the previous section, we assumed vehicles use a fixed traffic assignment method that minimizes total travel distance and number of turning maneuvers, and consequently, delay formulations do not depend on the traffic volume. However, these assumptions lose their applicability as trip demands grow and congestion occurs in the network. In these scenarios, drivers will still seek the route that provides them with the shortest travel time, but this might no longer be the one that minimizes the number of turning maneuvers performed and total travel distance. When no driver can improve his/her situation, the system has reached the user equilibrium (Wardrop, 1952). In this section we employ a static traffic assignment method to compare again how different grid networks (TW, TWL, and OW) behave under uniform demand loads that might create inhomogeneous congestion patterns (Diog et al., 2013). Simulations have two main advantages over the analytical formulations: a) they describe the behavior of trips when the network is congested; and, b) they capture the travel time as a function of the traffic load in the different links and intersections.

The traffic assignment model we employ is detailed in Ortigosa and Menendez (2014) and it is based on Leblanc et al. (1975) implementation using the Frank and Wolfe algorithm (Frank and Wolfe, 1956). To overcome the asymmetry of the problem, intersections are modeled employing 16 dummy links. For each one of them representing an intersection movement (e.g. left turn), a differentiable delay curve is created and adjusted to the Highway Capacity Manual 2010 (HCM-2010) formulation (HCM, 2010).

All the streets are modeled with the same characteristics: two lanes (one in each direction in the case of TW and TWL; the two in the same direction in the case of OW), and a saturation flow per lane of $1,800 \mathrm{veh} / \mathrm{h}$-lane. Every intersection node contains a traffic signal. A 60 second cycle length is assumed with 2.5 seconds of lost time per phase change. At intersections on TW networks, four-phase signals are used: two dedicated left-turn phases ( 2.5 seconds each) and two through/right-turn phases (22.5 seconds each). A dedicated left-turn pocket is also assumed to exist at these locations. At intersections on OW and TWL networks, two-phase signals are used: one for each perpendicular direction of movement (27.5 seconds each).

In our experiments we analyze networks of different sizes (number of intersection nodes per side), and we apply different levels of demand, from $T=0.01$ to $T=0.25$ trips between each two demand nodes 
(see Table 1). The total number of trips is not only function of the demand level but also of the size of the network, e.g. a 16x16 network will have 418 times more trips than a $4 \times 4$ network.

Table 1. Different networks analyzed.

\begin{tabular}{ccccc}
\hline $\begin{array}{c}\text { Nodes per } \\
\text { side }\end{array}$ & $\begin{array}{c}\text { Total } \\
\text { number of } \\
\text { nodes }\end{array}$ & $\begin{array}{c}\text { Total number of } \\
\text { links }\end{array}$ & $\begin{array}{c}\text { Area } \\
\left(\mathbf{k m}^{2}\right)\end{array}$ & $\begin{array}{c}\text { Range of total } \\
\text { number of trips }\end{array}$ \\
\hline 4 & 16 & 24 & $0.09-0.36$ & $5.5-138.0$ \\
6 & 36 & 60 & $0.25-1.00$ & $35.4-885.0$ \\
8 & 64 & 112 & $0.49-1.96$ & $124.3-3108.0$ \\
10 & 100 & 180 & $0.81-3.24$ & $322.2-8055.0$ \\
12 & 144 & 264 & $1.21-4.84$ & $694.3-17358.0$ \\
14 & 196 & 364 & $1.69-6.76$ & $1321.3-33033.0$ \\
16 & 256 & 480 & $2.25-9.00$ & $2299.2-57480.0$ \\
\hline
\end{tabular}

\subsection{Average trip length and average turns per trip}

Following a similar structure as in Section 2, the average trip lengths are now calculated for three different demand levels (See Figures $4 \mathrm{a}-\mathrm{c}$ ). These results are highly consistent with the analytical formulations (Figure 2a), and suggest that the presence of congestion does not considerably impact average distance traveled. Only for the demand level of $T=0.25$ and a network of size $n=16$ we do see how the average trip length of TWL increases, matching the one traveled in OW networks.

Average trip length a) $T=0.01$

b) $T=0.1$

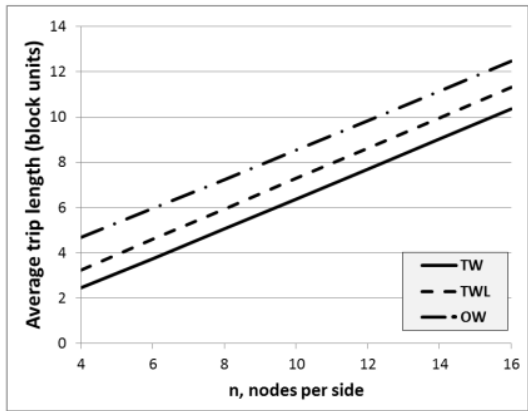

Average turns per trip

d) $T=0.01$

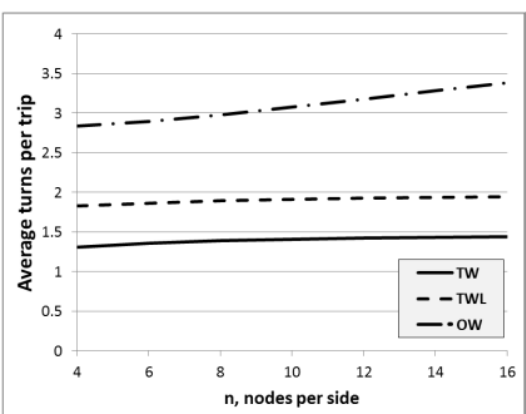

e) $T=0.1$

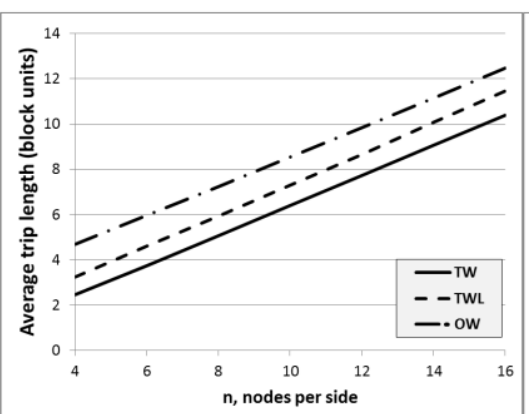

c) $T=0.25$

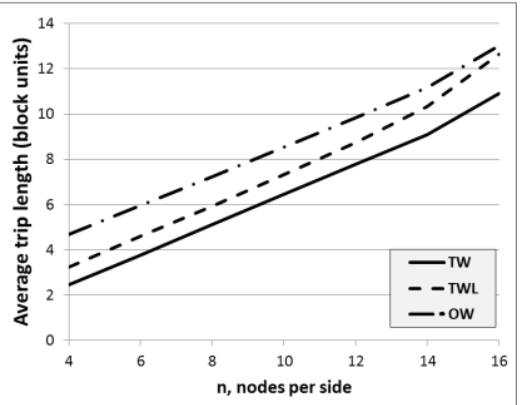

f) $T=0.25$
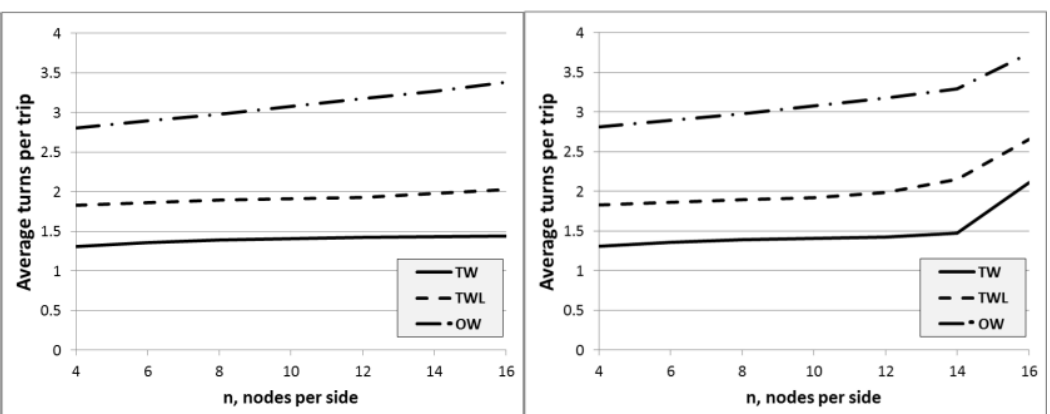

Fig 4. Average trip lengths and turns with the simulation model for demand levels of $T=0.01,0.1$, and 0.25 ; network sizes $n=4-16$ and links of $100 \mathrm{~m}$.

For each network type, the average number of turning maneuvers per trip appears to differ slightly more than the analytical counterparts. TW and TWL are fairly consistent with the analytical formulations, being close to 1.5 and 2 turns per trip, respectively. However, in reality, OW networks have higher turning rates than the analytical formulations show because there is no difference in delay 
between turning or through maneuvers since these movements share the same green phase. Therefore, if there are several routes with the same total distance and different number of turns, the vehicles will choose the one that is less congested. This serves to spread congestion more uniformly across the network in the simulation (these effects will be discussed in Section 4.2). Note that in reality vehicles might choose straighter routes because there might be some additional benefits, like signal coordination, but our model does not account for that. Finally, in Figure $4 \mathrm{f}$ we can see how for all cases the number of turns increases in comparison to lower demand levels. That means the system is so congested that vehicles explore other routes that might involve even more turns, but yield a lower total travel time.

\subsection{Travel time on networks}

Figure 3 shows the average travel times for the three networks according to the analytical formulations; in this section, we examine travel times using the simulation model which incorporates the impacts of congestion and demand-related delays at intersections. Figure 5 illustrates the travel times for different sizes, different levels of demand, and link lengths.

When the demand level is really low and the links are $100 \mathrm{~m}$ long (Figure 5a), we observe high consistency with the results of the analytical formulations shown in Figure 3a. In this scenario, TWL networks provide the shortest travel times when networks are small (i.e., $n$ is low); this is followed very closely by TW networks and finally OW networks. As the size of the networks increases, the difference between TWL and TW increases because the latter type is penalized by the additional signal phases required at the intersections. For every intersection, TWL and OW provide additional green time for movement due to less lost time (e.g. in this simulation, 5 extra green time seconds) and this results in lower average delays. Overall, this diminishes the performance of TW in comparison to the other network types when demand and network size increase.

When networks become more congested, we see that travel times increase in an exponential manner with network size. Also, we see how TWL and OW get closer until OW becomes more competitive than TWL for 16 nodes per side and demands greater than 0.1 . The most striking phenomenon occurs when networks are large $(n=16)$ and very congested (Figures $5 \mathrm{c}$ and $5 \mathrm{f}$ ) where TW networks are significantly better than the rest. Notice that these behaviors cannot be seen with the analytical formulations since they are due to the effects of congestion, specifically: the path redundancy of the networks, the heterogeneity of congestion, and the delay formulation employed. We examine in detail all these effects in Sections 4.2 and 4.3.

We observe a sudden increase in travel times when networks are larger than 14 nodes per side and demand level is 0.25 . According to the simulation results, more than $40 \%$ of the intersection approaches have V/C ratios larger than 1; explaining such an increase in travel times, and, making these results in heavy congestion somewhat unreliable. . 
Link length $=100 \mathrm{~m}$

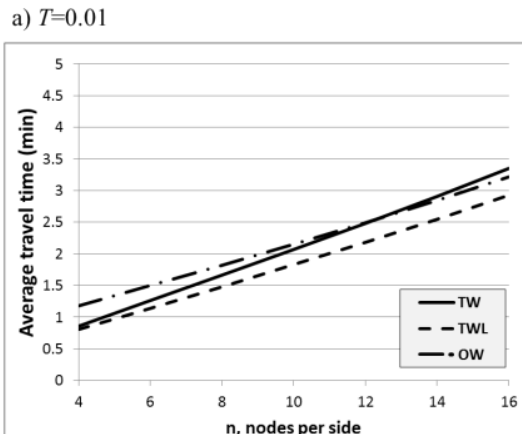

b) $T=0.1$

c) $T=0.25$
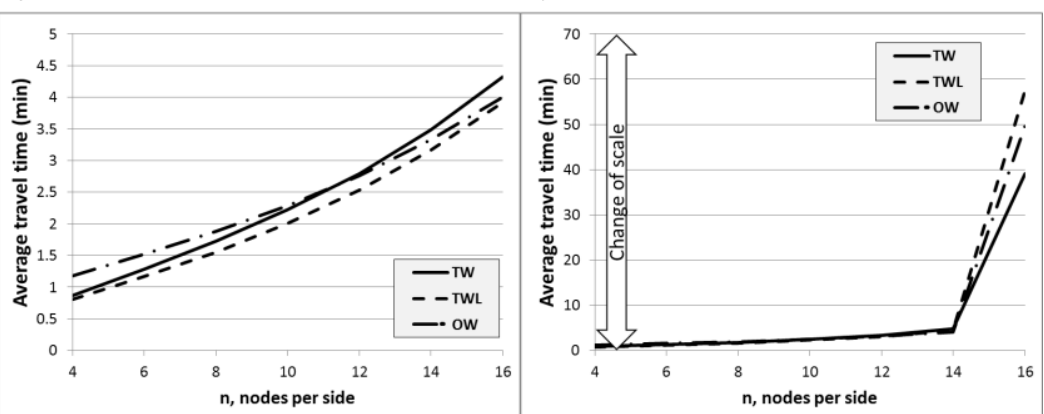

Link length $=200 \mathrm{~m}$

d) $T=0.01$

e) $T=0.1$

f) $T=0.25$
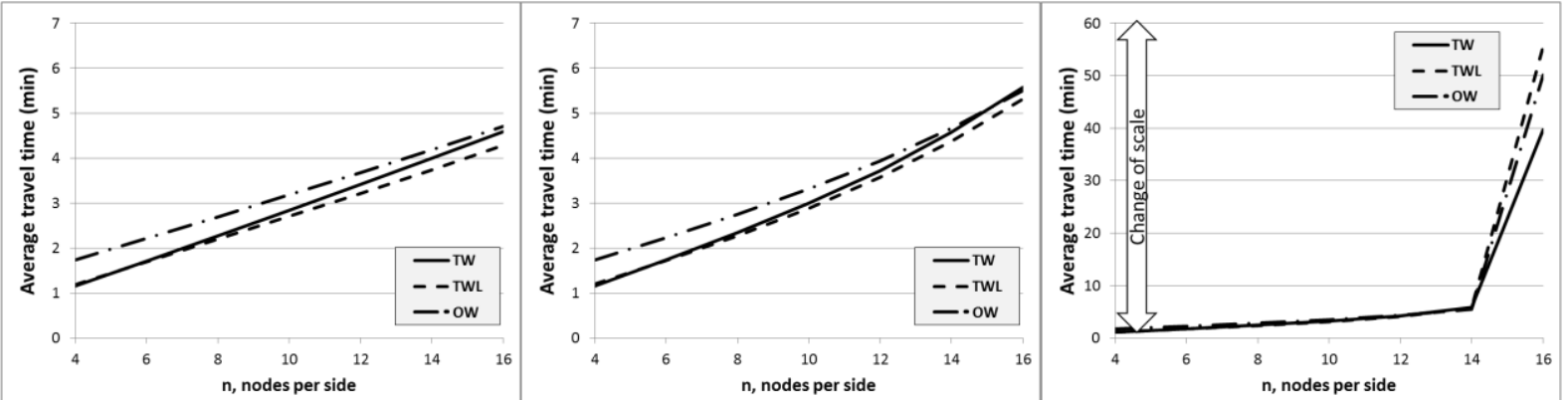

Fig 5. Average travel times with the simulation model for demand levels of $T=0.01,0.1$, and 0.25 , network sizes $n=4-16$ and links of $100 \mathrm{~m}$ and $200 \mathrm{~m}$.

TW networks offer the shortest routes with the least number of turns, but are penalized by the reducedcapacity at the intersections. However, as links become longer the ratio between travel time on the links and travel time at the intersections increases, and this allows the TW networks to become more competitive. This case is depicted on Figures 5d-f, which provide simulation results for links that are $200 \mathrm{~m}$ long. In this new scenario, the general trends repeat but we observe that the curve for TW travel times becomes lower (and the curve for the OW travel times becomes higher) than the case where links are $100 \mathrm{~m}$ long. For example, when networks are $n=4$, TW networks are better for all the demand levels. Networks with higher average trip lengths like OW are more penalized because travel time on links is higher.

\section{Analysis of results}

Seeing the previous results, and considering travel time as the most important variable, TWL networks provide the best performance in $83 \%$ of the cases (70 out of 84 ) analyzed (Figure 6 ), TW in $12 \%$ of the cases (10 out of 84 ), and OW only in $5 \%$ of the cases (4 out of 84 ). In this section we study in detail why these results are obtained to unveil the strengths and weaknesses of each network. 

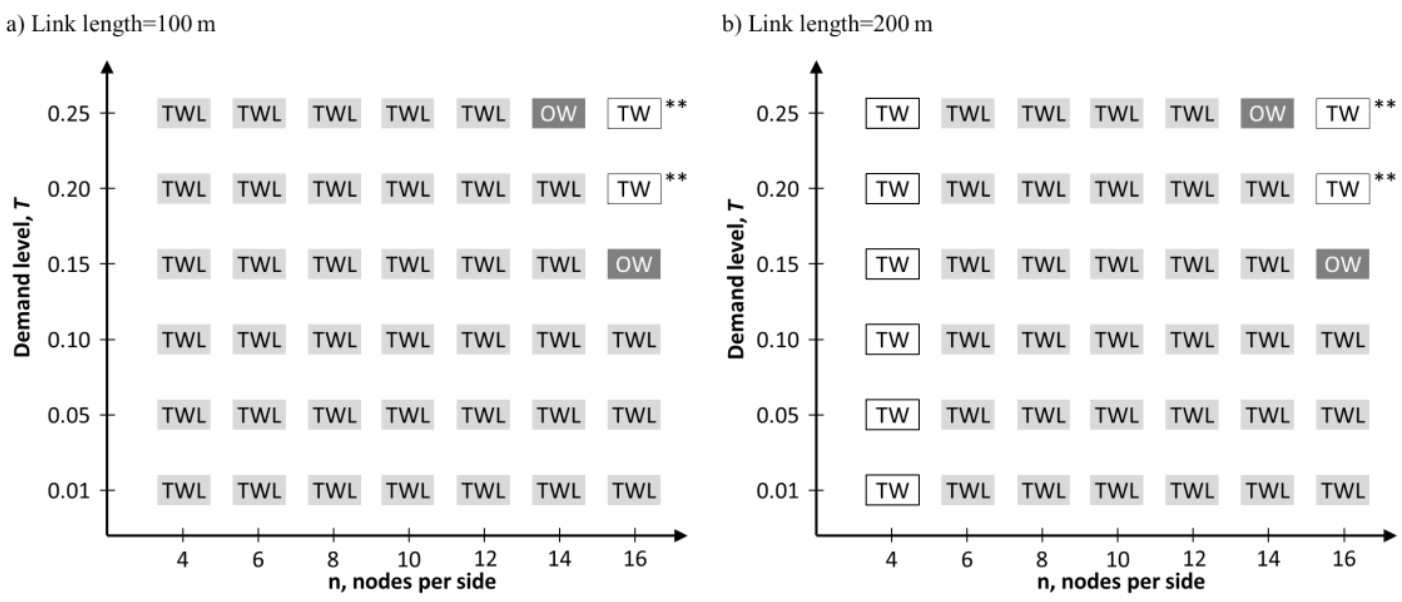

Fig 6. Lowest travel time network type for demand levels of $T=0.01-0.25$, network sizes $n=4-16$ and links of 100 $\mathrm{m}$ and $200 \mathrm{~m}$.

\subsection{Comparison between analytical approach and simulation}

As we discussed, analytical formulations are suitable for uncongested scenarios whereas simulations describe better congestion situations. In spite of that, the analytical formulations are very accurate in many scenarios, especially when it comes to average travel distance. Figure 2a (analytical) and Figures $4 \mathrm{a}-\mathrm{c}$ (simulation) show that the average trip length is certainly the metric that is less influenced by congestion; hence, analytical formulations are suitable to describe distance traveled in many different demand and network size scenarios. Moreover, average turns per trip are quite consistent for TW and TWL networks, except for very congested cases. OW simulations, however, show more turns than analytical formulations because in the case of equal length routes, turning or going through both cause the same delay.

Figure 3 and Figure 5 present travel times for analytical formulations and simulations, respectively. This is the most sensitive indicator of the congestion level. When demands are very low, the simulation results (Figure 5a) are very similar to the results obtained from the analytical formulations (Figure 3a). However, as trip demand increases, travel time magnitudes increase as well, and the fit between simulations and the analytical formulations deteriorates. Figure 3 was calculated assuming that V/C ratios on (18), (19), and (20) were 0 considering the system very uncongested. It is then reasonable that results differ from the simulations when congestion arises. We have calculated analytically the average V/C ratios for the other levels of demand and for each network type, and applied them to expressions (18), (19) and (20). On average, analytical formulations provide rather reliable results (Table 2 shows the errors between the analytical approach and the simulation for TW networks); and only in cases with high demands and network sizes we observe large divergences (bold values Table 2). Obviously, these results cannot be used for detailed traffic assignment purposes or to compare two network configurations because errors are computed in absolute values. Nevertheless, these analytical formulations might be very useful and save a lot of computational efforts for more general and macroscopic analyses. 
Table 2. Absolute values of relative (total) error between analytical formulations and simulations for average travel time on TW networks in units of percent difference (seconds).

\begin{tabular}{ccccccc}
\hline $\begin{array}{c}\text { Nodes per } \\
\text { side }\end{array}$ & $\mathbf{0 . 0 1}$ & $\mathbf{0 . 0 5}$ & $\mathbf{0 . 1}$ & $\mathbf{0 . 1 5}$ & $\mathbf{0 . 2}$ & $\mathbf{0 . 2 5}$ \\
\hline 4 & $9.0 \%(5 \mathrm{~s})$ & $8.7 \%(5 \mathrm{~s})$ & $8.4 \%(5 \mathrm{~s})$ & $8.2 \%(5 \mathrm{~s})$ & $7.9 \%(4 \mathrm{~s})$ & $7.6 \%(4 \mathrm{~s})$ \\
6 & $7.1 \%(6 \mathrm{~s})$ & $6.5 \%(5 \mathrm{~s})$ & $5.7 \%(5 \mathrm{~s})$ & $4.9 \%(4 \mathrm{~s})$ & $4.1 \%(3 \mathrm{~s})$ & $3.4 \%(3 \mathrm{~s})$ \\
8 & $6.4 \%(7 \mathrm{~s})$ & $5.0 \%(5 \mathrm{~s})$ & $3.4 \%(4 \mathrm{~s})$ & $1.8 \%(2 \mathrm{~s})$ & $0.2 \%(<1 \mathrm{~s})$ & $1.3 \%(1 \mathrm{~s})$ \\
10 & $5.9 \%(8 \mathrm{~s})$ & $3.4 \%(4 \mathrm{~s})$ & $0.4 \%(1 \mathrm{~s})$ & $2.5 \%(3 \mathrm{~s})$ & $5.3 \%(7 \mathrm{~s})$ & $8.0 \%(11 \mathrm{~s})$ \\
12 & $5.3 \%(8 \mathrm{~s})$ & $1.2 \%(2 \mathrm{~s})$ & $3.7 \%(6 \mathrm{~s})$ & $8.4 \%(14 \mathrm{~s})$ & $\mathbf{1 2 . 8 \% ( 2 1 s )}$ & $\mathbf{1 7 . 0 \% ( 2 9 s )}$ \\
14 & $4.6 \%(9 \mathrm{~s})$ & $1.7 \%(3 \mathrm{~s})$ & $9.1 \%(17 \mathrm{~s})$ & $\mathbf{1 5 . 8 \% ( 3 1 s )}$ & $\mathbf{2 2 . 0 \% ( 4 5 s )}$ & $\mathbf{3 7 . 2 \% ( 7 8 s )}$ \\
16 & $3.8 \%(8 \mathrm{~s})$ & $5.4 \%(12 \mathrm{~s})$ & $\mathbf{1 5 . 6 \% ( 3 5 s )}$ & $\mathbf{2 4 . 8 \% ( 5 8 s )}$ & $\mathbf{1 4 7 . 4 \% ( 3 6 4 s )}$ & $\mathbf{7 9 4 . 8 \% ( 2 0 8 1 s )}$ \\
\hline
\end{tabular}

\subsection{Heterogeneity of congestion and redundancy}

In transportation networks, path redundancy is desired because drivers have alternatives when some routes are blocked. Moreover, redundancy may imply reliability and resilience, both, desirable qualities for transportation networks. Given any two points on a TW, TWL, or an OW network, there is only one route that simultaneously minimizes both distance and the number of turns; thus, no flexibility in route choice exists (Figure 8a). If the turn condition is relaxed and only the distance minimization is considered, the routing flexibility changes based on the network type. In a TW network, the number of shortest paths between two intersections nodes is $\left(\begin{array}{c}t_{l} \\ d_{s}\end{array}\right)$, where $t_{l}$ is the total length of the trip in blocks, and $d_{s}$ is the minimum orthogonal component in blocks. As an example, in Figure 8a, there will be 6 different minimum distance routes between demand nodes $i$ and $j$ (or between intersections nodes $n_{i}$ and $n_{j}$ ). At the other extreme lies TWL networks, which offer no flexibility at all. Consider Figure $8 \mathrm{a}$-all alternative routes require more travel distance. OW networks lie somewhere in between these two extremes: some OD pairs can be served by only a single route while others have multiple routes with the same (minimum) travel distance (see Figure 8a).

Let us now illustrate the path redundancy of the three networks with an example employing the simulation scheme (Figure $8 \mathrm{~b}$ ). In an empty network, we load different demand levels from point $\mathrm{A}$ to point $\mathrm{B}$, and we see how the traffic assignment routes them through the network. Notice that $T$ here is much larger than before because we are only considering one OD pair in an empty network. TW networks are able to spread flows in a very efficient manner, so much that when the level of demand is the highest, the links become much less congested than in the other networks. TWL networks are especially rigid, and only when the system is very congested flows spread and even then, they do so only slightly. Our simulation model does not consider spillbacks, but this is a phenomenon that can be very critical for TWL since all the demand is channelized only through one route. If this route is blocked, the increase in travel times might be critical because the alternatives suppose longer distance and many turns. OW networks keep a medium spread that is fairly constant with the different levels of demand. It is worth pointing out that this particular A to B combination allows more shortest path routes between the two points than other combinations in the OW network. In other words, other A to $\mathrm{B}$ combinations might not have that many available shortest paths, so congestion would be more concentrated. 
a)
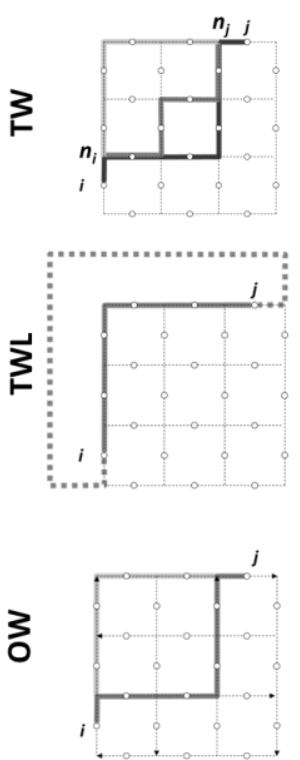

b)

$T=100$
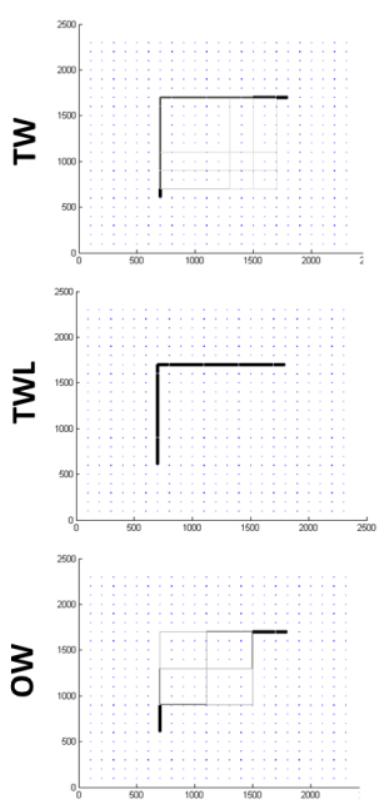

$T=500$
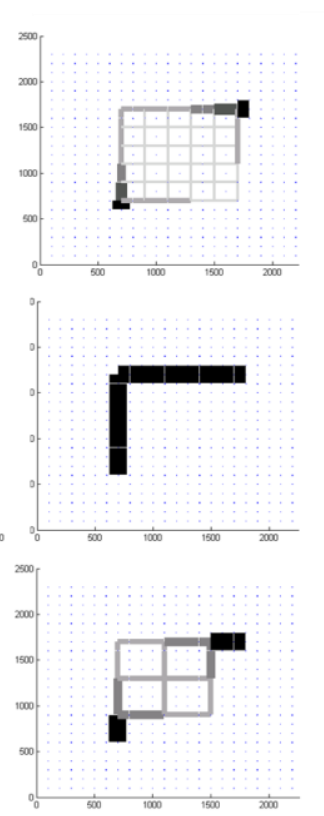

$T=1000$
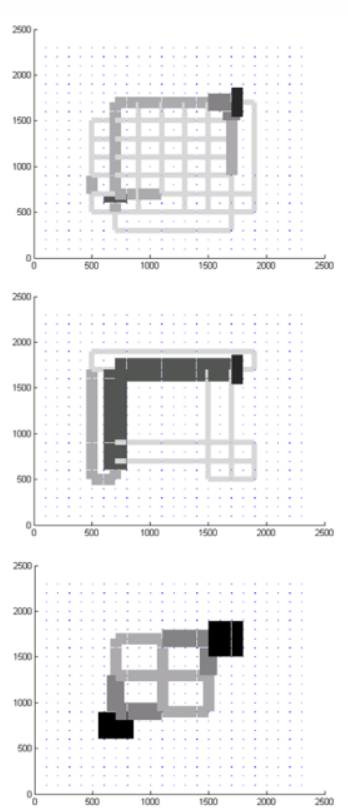

Fig 7. a) Examples of path redundancy in TW, TWL, and OW networks; b) Flow spread between an origin and destination with different levels of demand.

Analyzing the simulations results, on average, the V/C ratios of the networks are lower for TW than for TWL and OW. Especially in very congested cases, when networks are very large in size (e.g.n=16) and demand levels are also large (e.g. $T=0.20$ and 0.25 ), TW networks also have a lower percentage of links with V/C ratios exceeding 1 than the other two network types. Simulation results might not be realistic because such levels of congestion might involve spillbacks, and the gridlock of the system. Nevertheless they are useful to explain general patterns. For example, that the decrease on total travel time of TW networks when congestion is high happens because they are able to spread congestion more efficiently, and this occurs because they offer more redundant shortest path routing.

\subsection{TWL vs. OW networks under congestion}

Another effect that we observe in simulations results is how TWL and OW networks tend to have a similar average travel time when networks are congested; and even in some cases, OW networks can provide shorter travel times. One of the reasons for that is the variability on V/C ratios that TWL networks present. Even though the average V/C ratio is slightly lower for TWL compared to OW, the variance is much higher, i.e. congestion levels are more heterogeneous. Since delay formulations are not linear, a higher heterogeneity in V/C ratios will cause a higher delay. Another reason is the type of delay functions we employ at intersections. The HCM 2010 formulations are dependent on the total saturation flow of each approach (in addition to the V/C ratio, green time and cycle length). Because both lanes are used to serve a single direction in the OW network, the saturation flow at the intersection is twice that of the TWL network $(3,600 \mathrm{veh} / \mathrm{h}$ as opposed to $1,800 \mathrm{veh} / \mathrm{h})$. Hence, for any given V/C ratio, the HCM 2010 methodology provides a much higher delay for the approach with a lower saturation flow (TW and TWL) than for the approach with a higher saturation flow (OW). 


\section{Conclusions}

In this paper we studied different configurations of streets in finite urban grids: two-way streets (TW), two-way streets with prohibited left turns (TWL), and one-way streets (OW). This is a subject of much controversy and our goal was to provide an in-depth study from a traffic perspective that might help urban planners when deciding on one structure vs. another one. We assumed a perfect finite grid network emulating an urban environment with uniform demand, and studied the behavior of traffic under two main scenarios: uncongested networks (analytical formulations), and congested networks (simulation). The results presented here provide a general overview of how the different street layouts behave and what factors urban planners and traffic engineers should consider.

When networks are not congested, i.e. traffic volumes are low, drivers are likely to select the route that provides the shortest travel distance, which is independent of the behavior of others vehicles. In this context, analytical formulations can be applied providing very useful and compact expressions to calculate total distance traveled, average trip length, average turns per trip, and average travel time (Section 2 and Section 4.1). Analytical formulations are not only useful because of their simplicity, but because they allow us to identify behavioral trends. TW networks achieve the lowest average trip length, whereas on average, a trip is one block longer for TWL networks and two blocks longer for OW networks. Regarding average turns per trip, TW networks require approximately 1.5 turns per trip, whereas TWL and OW networks require 2 and 2.5, respectively. Nonetheless, despite providing the least distance traveled and lowest number of turns, TW networks are penalized when it comes to travel time because the capacity per movement at intersections is the smallest out of the three networks. As Figure 3 shows, in uncongested scenarios, TWL networks provide the better compromise between distance traveled and delay at intersections.

Congestion increases travel times on the minimum distance routes, forcing drivers to seek alternative routes to lower their travel times. This behavior cannot be replicated with analytical formulations. Hence, we employed a static traffic assignment program (Ortigosa and Menendez, 2014) that approximates the system to the user equilibrium. For medium block size length (100 m), TWL is still the best configuration for the majority of scenarios because of the better balance between distance traveled and delay at intersections. However, when demand is high and networks are congested, TWL sometimes lose that advantage because they are not able to spread congestion as well as TW and to a smaller degree, OW (see Section 3.2). At some point, in very congested scenarios, OW networks gain advantage over TWL networks due to the heterogeneity of congestion and the delay formulations employed (Section 4.3). TW networks provide the best results in very congested scenarios (Figure 5) because of the availability of routes that allows congestion to spread more evenly. Nonetheless, such high congestion levels are not desirable in urban street networks (Daganzo, 2007). When streets are longer, as the case here analyzed $(200 \mathrm{~m})$, the distance traveled has a greater effect on the travel time. In this scenario, TW networks provide the best results for small networks $(n=4)$ and for very congested cases ( $n=16$ and $T=0.2)$ because they provide the shortest average trip length and also the best spread of congestion. OW networks are the most penalized because, on average, cars travel two blocks more than in TW networks.

Overall, there is not a unique network which fits best all scenarios; each presents particular advantages and disadvantages. After carrying out extensive evaluation under various scenarios, we summarize the main characteristics of each network type as follows: 
- Two-way networks (TW) provide the shortest distance traveled and are able to spread congestion better due to the redundancy of shortest path routes. They are, however, penalized by the reduced capacity offered at the more complicated intersections. This street configuration is suitable for small and uncongested networks, and for networks with long streets to avoid queue spillback concerns. They also seem to be very efficient in extremely congested cases, but these results might not be realistic due to the large amount of oversaturated links.

- Two-way networks with prohibited left turns (TWL) present the best compromise between distance traveled and capacity at intersections. That makes them suitable for the widest range of scenarios including both small and large networks, with short and long streets and low to moderate congestion. The main weakness of this network type is the rigidity of shortest path routes. This causes an inhomogeneous distribution of congestion and reduced resilience in cases with high levels of congestion. However, for networks with demand patterns that result in uniform congestion distributions across all links (e.g. see Diog et al (2013)), this might not be as large of a concern. Still, the impact of queue spillbacks might reduce the applicability of TWL networks in mediumhigh congested scenarios in favor of OW and TW networks.

- One-way networks (OW) are only the best option in a few scenarios. Their main disadvantage is that they force vehicles to travel the longest distances on average. These additional travel distances create a large penalty to overcome in networks with long streets. However, in networks with short streets and very high levels of congestion OW networks offer a better spread of the congestion than TWL networks, and are less likely to present spillbacks than TW networks.

We have considered three main variables: network size, level of demand, and link length. The first two affect the total trip demand of the system, whereas the later affects, basically, the distance traveled. For low trip demand scenarios and short links, the best configuration is TWL networks, shown both with analytical formulations and simulations. For low trip demand scenarios but long streets, TW networks, provide a slightly lower travel time (also shown in both analytical formulations and simulations). When the network is more congested (more demand and larger size) and links are short, TWL networks are the best option followed by OW ones. Our results show that TWL networks are superior in most of the scenarios; however, some of this advantage might be lost when taking into account queue spillbacks in highly congested scenarios. Finally, for congested scenarios in long link networks, TWL keep providing the best performance (shown again in both analytical formulations and simulations). However, the low redundancy of TWL might make TW networks more suitable because they spread congestion better.

Future research studies should focus on the dynamics of traffic for the different networks. In this work, spillbacks were not considered, but this is worth studying in the future to evaluate how TWL can be affected by them given they are the least redundant networks (Section 4.2). In this paper we considered a uniform demand pattern and a homogeneous grid design equal for the three configurations. In the future, the analysis could be done differently: which configuration fits better a given demand pattern? And also, study if some grid structures (e.g. hierarchy of streets, public transportation presence) could influence our choice of network. 


\section{References}

Abdel-Aty, M., Abdalla, M.F., 2004. Modeling drivers' diversion from normal routes under ATIS using generalized estimating equations and binomial probit link function. Transportation 31(3), 327-348.

Boyles, S.D., Rambha, T., Xie, C., 2014. Equilibrium analysis of low-conflict network designs. Submitted to Transportation Research Record: Journal of the Transportation Research Board.

Cervero, R. (2001). Induced Demand: An Urban Metropolitan Perspective. UC Berkeley: University of California Transportation Center. Retrieved from: https://escholarship.org/uc/item/5pj337gw.

Daganzo, C.F., 2007. Urban gridlock: Macroscopic modeling and mitigation approaches. Transportation Research Part B: Methodological 41(1), 49-62.

Doig, J., Gayah V., Cassidy, M., 2013. Inhomogeneous flow patterns in under-saturated road networks and implications for the MFD. Transportation Research Record: Journal of the Transportation Research Board $2390,68-75$.

Eichler, D., Bar-Gera, H., Blachman, M., 2013. Vortex-based zero-conflict design of urban road networks. Networks and Spatial Economics 13(3), 229-254.

Estrada, M., Roca-Riu, M., Badia, H., Robusté, F., Daganzo, C.F., 2011. Design and implementation of efficient transit networks: Procedure, case study and validity test. Transportation Research Part A: Policy and Practice 45(9), 935-950.

Frank, M., Wolfe, P., 1956. An algorithm for quadratic programming. Naval Research Logistics Quarterly 3, $95-110$.

Gayah, V., Daganzo, C., 2012. Analytical capacity comparison of one-way and two-way signalized street networks. Transportation Research Record: Journal of the Transportation Research Board 2301, 76-85.

HCM, 2010. Highway Capacity Manual. 5th Edition. Transportation Research Board.

Hocherman, I., Hakkart, S., Bar-Ziv, J., 1990. Safety of one-way urban streets. Transportation Research Record: Journal of the Transportation Research Board 1270, 22-27.

LeBlanc, L. J., Morlok, E.K., Pierskalla, W.P., 1975. An efficient approach to solving the road network equilibrium assignment problem. Transportation Research 5, 309-318.

Lyles, R., Faulkner, C., Syed, A., 2000. Conversion of streets from one-way to two-way operation. Final report for the Michigan Department of Transportation.

Mannering, F., Kim, S., Barfield, W., Ng, L., 1994. Statistical analysis of commuters' route, mode, and departure time flexibility. Transportation Research Part C 2(1), 35-47.

Meng, L.K., Thu, S., 2004. A microscopic simulation study of two-way street network versus one-way street network. Journal of the institution of engineers 44(2), 111-122.

Ortigosa, J., Menendez, M., 2014. Analysis of quasi-grid urban structures. Cities 36, 18-27.

Stemley, J., 1998. One-way streets provide superior safety and convenience. ITE Journal 68(8), 47-50.

Tindale, S., Hsu., P., 2005. Crash data and signal coordination: A one-way pair case study. Journal of Safety Research 36(5), 481-482.

Walker, G., Kulash, W., McHugh, B., 2000. Downtown streets: Are we strangling ourselves on one-way networks? Transportation Research Circular 501, 18.

Wardrop, J.G., 1952. Some theoretical aspects of road traffic research. Proceedings - Institution of Civil Engineers - Part 2: Research and Theory 1, 325-378. 